\title{
LITO - A Logic For Indeterminate Temporal Objects
}

\author{
Ed Porto BEZERRA ${ }^{1}$, Ulrich SCHIEL ${ }^{2}$, Bernardo LULA JR ${ }^{2}$ \\ ${ }^{1}$ Departamento de Informática, UFPB/CCEN, João Pessoa-PB, \\ ${ }^{2}$ Departamento de Sistemas e Computação, UFCG/CCT, Campina Grande-PB, \\ e-mail:edporto@di.ufpb.br, (ulrich,lula)@dsc.ufcg.edu.br
}

\begin{abstract}
In order to obtain a suitable model of the universe of discourse of some information systems, it is important to allow the description of imprecise objects with temporal indeterminacy. These objects are called indeterminate temporal objects. This paper presents a formalism, called LITO (Logic of Indeterminate Temporal Objects), for the representation, querying and modification of such objects.
\end{abstract}

\section{Introduction}

In certain applications, it is fundamental to consider historical objects. Moreover, the information about the objects of the application frequently cannot be completely determined. However, the current computer information systems (including the database management systems) do not feature resources to represent, retrieve and modify such objects, here called indeterminate temporal objects.

Researches about incomplete information in information systems exist since the 1970's [11]. However, their integration with database systems is restricted to the null value concept for an attribute. Similarly, research about maintenance of that object was not so much undertaken [5]. Both formalisms and temporal query languages present partial solutions to representing and manipulating indeterminate temporal objects.

The main objective of this paper is to show a formalism that gives definitions about representation and manipulation of indeterminate temporal objects in indeterminate temporal database (ITDB). More precisely, we herein introduce LITO (Logic of Indeterminate Temporal Objects), a formalism that allows for retrieval query, logical query, insertion, logical deletion, refinement and update of indeterminate temporal objects. Futhermore, a computer system was implemented in LPA-Prolog [9] to show how LITO can be applied in practice.
Section 2 shows the theoretical foundation for temporal database and indeterminacy. Related works are treated in section 3. Section 4 describes LITO. The last section encompasses final comments.

\section{Temporal Database and Indeterminacy}

We consider time as a discrete, infinite, and totally ordered set of points called instants. Given two instants $t_{1}$ and $t_{2}$, the set of all instants between them, including $t_{1}$ and $t_{2}$, is called an interval and is denoted as $\left[t_{1}, t_{2}\right]$. A set of one or more disjoint intervals is called a time element.

A temporal database is a database where time elements are associated to some data elements (attributes and objects). These time elements determine the valid time, that is, they specify the time when the corresponding information holds in the real world modeled by the database [7]. Data elements as well as time elements may be well determined or more or less indeterminate. For data elements, this corresponds to situations where "we know exactly what" or "we do not know exactly what" while, for time elements, the meaning is that "we know exactly when" or "we do not know exactly when".

A database models a part of the real world called universe of discourse. If the available information is complete and precise, then there is a clear correspondence between the database and the universe of discourse. When the knowledge about the real world is incomplete, several scenarios are possible, but it is not known which of them represents the real status of the world. A database containing incomplete information implicitly represents a set of possible worlds.

Possible-world scenarios are supported by the socalled open-world assumption (OWA) [8]. Under the usual closed-world assumption, facts not explicitly stored nor deducible from true facts are considered false. Under the OWA, a statement is considered false only if it is explicitly stored as false or if its negation can be logically derived from the database. The information neither explicitly present in the database nor derivable from explicit information is considered unknown; the 
information contained in one but not all possible worlds is considered possible. The information contained in all possible worlds is considered true while information not present in any possible worlds is considered false.

Temporal indeterminacy characterizes situations where an instant is not perfectly defined but is described instead as an interval of possible values. For instance, during 2001 means, with a granularity of day, "some day between 1/1/2001 and 31/12/2001".

An indeterminate interval is an interval delimited by at least one indeterminate instant [7]. A temporal database where both data elements and time elements can be indeterminate is called an Indeterminate Temporal Database (ITDB).

\section{Related Work}

The formalization of a theory for modification operations in temporal database does not exist. The same happens for manipulation operations in ITDB. TSQL2 language [14] only considers retrieval operation to obtain registers in valid timetables, i.e., it does not consider modification operations. This limitation compromises the language's usability for real situations. TSQL2 provides a form of representing indeterminate instants and other way to represent indeterminate periods [4]. For instance, the notation 10/5 29/5 is used to denotes an instant between 10 and 29 of May, and the notation [1/3/04 31/3/04 $1 / 6 / 04 \sim 30 / 6 / 04]$ is used to denotes a interval with indeterminate endpoints.

The Logic of Incomplete Knowledge Base [10] is an adequate method to represent objects in OWA. Despite, it treats neither temporal indeterminacy nor modification of objects.

The majority of proposed query languages to temporal database extend only SQLs select command. Elsewhere, SQL/Temporal [12] and TSQL2 [14] are the first approaches considering temporal modification. Unfortunately, they evolve only periods with determinate time.

\section{A Formalism for Indeterminate Temporal Objects}

This formalism aims to support a formal syntax for definition and manipulation operations ITDBs. It is out of the papers scope to present the complete formalism that can be found in [1]. So, this section shows the essentials of the formalism.

\subsection{The Language}

LITO is formed by a triple $<L, A, R>$ where $L$ is a language, $A$ is the set of axioms that characterizes the temporal and modal operators, and $R$ is the set of rules to be used for deduction of new facts (modus ponens) and to process queries on the objects.

4.1.1 Alphabet: The alphabet of LITO is the minimal set of basic symbols

Logic symbols:

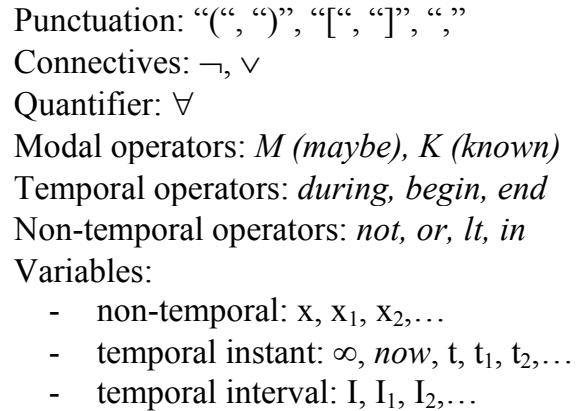

The basic modal operator $M$ (maybe) describes indeterminate information and the basic modal operator $K$ (known) characterizes information stored in the ITDB. These operators have the same signification used in modal logic [6] and incomplete knowledge base logic [10], respectively. Non-temporal operators describe indeterminate non-temporal information, i.e., in this paper, they describe imprecise, unknown, and negative information, according to [1]. Temporal operators will be explained in section 4.2

\section{Non-logic symbols:}

- constants:

- temporal: *.

- non-temporal: *, none and other character strings.

- Predicative symbols: Time, $<,=, \mathrm{P}, \mathrm{P}_{1}, \mathrm{P}_{2}, \ldots$

The temporal constant $*$ is necessary for representing both temporal indeterminate values and non-temporal indeterminate values, while the non-temporal constant none denotes a non-existent unknown information.

The set of predicative symbols is open. Each such symbol (except the three predicate symbols Time, $<$, and $=)$ must begin with the letter P. The symbol Time denotes the valid time of an object. Its first argument is a nontemporal variable that identifies the object. The second argument is a temporal term (section 4.1.2).

4.1.2 Terms: Terms are expressions of the language L, used to denote information capable of being stored in ITDB. They are classified in non-temporal terms, temporal instant terms, and temporal interval terms. The L's set of terms is the minimal set satisfying the following conditions:

Non-temporal terms:

- Each non-temporal constant is a non-temporal term;

- Each non-temporal variable is a non-temporal term; 
- if $\mathrm{x}$ is a non-temporal variable, and $\mathrm{Tx}, \mathrm{Tx}_{1}$ and $\mathrm{Tx}_{2}$ are non-temporal terms, then $\operatorname{or}\left(\mathrm{Tx}_{1}, \mathrm{Tx}_{2}\right), \operatorname{not}(\mathrm{Tx})$, and $l t \mathrm{x}$ are non-temporal terms.

Definite temporal instant and indefinite temporal instant terms:

- the temporal constants $*$ and $\infty$ are indefinite temporal instant terms;

- each temporal instant variable is a definite temporal instant term;

- if TI is a temporal interval term, then during TI is an indefinite temporal interval term;

- if TI is a temporal interval term, then begin TI and end TI are indefinite temporal instant terms;

The distinction between indefinite and definite terms occurs because the order relation $(<)$ is not applicable to terms * and during TI. These terms do not precisely define the temporal instant represented. The order relation is applicable only to definite temporal instant terms. The general denomination "temporal instant term" is used to describe both types of temporal instant terms.

Temporal interval terms:

- each temporal interval variable is a temporal interval term;

- if $\mathrm{Tt}_{1} \mathrm{e} \mathrm{Tt}_{2}$ are temporal instant terms, then $\left[\mathrm{Tt}_{1}, \mathrm{Tt}_{2}\right]$ is a temporal interval term.

4.1.3 Formulas: Formulas are expressions in the language L, used to denote facts in the domain of the represented application. A well-formed formula (wff) is recursively defined as follows:

- if $\mathrm{x}$ is a non-temporal variable, $\mathrm{Tx}, \mathrm{Tx}_{1}$ and $\mathrm{Tx}_{2}$ are nontemporal terms, and TI is a temporal interval term, then $\mathrm{Tx}_{1}=\mathrm{Tx}_{2}, \mathrm{P}(\mathrm{x}), \mathrm{P}(\mathrm{x}, \mathrm{Tx}), \operatorname{Time}(\mathrm{x}, \mathrm{TI})$ are wffs;

- if $\mathrm{F}$ and $\mathrm{G}$ are wffs, then $\neg \mathrm{F}, \mathrm{F} \vee \mathrm{G}, M \mathrm{~F}$ and $K \mathrm{~F}$ are wffs;

- if $\mathrm{F}$ is a wff and $\mathrm{v}$ is a non-temporal variable, a temporal instant or some temporal interval, then $\forall \mathrm{v}(\mathrm{F})$ is a wff;

- if $\mathrm{Tt}_{1}$ and $\mathrm{Tt}_{2}$ are definite temporal instant terms, then $\mathrm{Tt}_{1}=\mathrm{Tt}_{2}$ and $\mathrm{Tt}_{1}<\mathrm{Tt}_{2}$ are wffs;

- if $\mathrm{TI}_{1}$ and $\mathrm{TI}_{2}$ are temporal interval terms, then $\mathrm{TI}_{1}=\mathrm{TI}_{2}$ is a wff.

Note that the predicative symbol $\mathrm{P}$ can express both a class of objects and the attributes of this class. In the first case, $\mathrm{P}$ is the name of class and $\mathrm{x}$ contains the identifier of the class' objects. An attribute of the object $x$ will be represented by a binary predicative symbol $\mathrm{P}(\mathrm{x}, \mathrm{Tx})$ such that $\mathrm{Tx}$ represents the attribute value and $\mathrm{P}$ is its name.

4.1.4 Definitions: The symbols $\exists, \wedge$, and $\rightarrow$ are defined as in classical logic. For definite temporal instant terms we define.

1. $\mathrm{Tt}_{1} \in\left[\mathrm{Tt}_{2}, \mathrm{Tt}_{3}\right] \Leftrightarrow$ def $\left(\mathrm{Tt}_{2} \leq \mathrm{Tt}_{1}\right) \wedge\left(\mathrm{Tt}_{1} \leq \mathrm{Tt}_{3}\right)$

This relation of pertinence characterizes temporal interval as a linear and connected set.

\section{2. $\mathrm{I}=\left[\mathrm{t}_{1}, \mathrm{t}_{2}\right] \Leftrightarrow_{\text {def }} \mathrm{t}_{1}=$ begin $\mathrm{I} \wedge \mathrm{t}_{2}=$ end $\mathrm{I}$}

An interval I represented by $\left[\mathrm{t}_{1}, \mathrm{t}_{2}\right]$ is delimited by definite temporal instants $t_{1}$ and $t_{2}$, where $t_{1}$ is the initial instant of I and $t_{2}$ is its final instant.

3. $\mathrm{I}_{1} \subseteq \mathrm{I}_{2} \Leftrightarrow{ }_{\text {def }}$ (begin $\mathrm{I}_{2} \leq$ begin $\left.\mathrm{I}_{1}\right) \wedge\left(\right.$ end $\mathrm{I}_{1} \leq$ end $\left.\mathrm{I}_{2}\right)$

The relation "is contained in or is equal to" applied to intervals is defined according to their limits.

Definitions 8 and 9 use the linear and discrete structures of the time and they respectively express the successor and predecessor operators for temporal instants.

4. $\mathrm{t}_{2}=\operatorname{succ} \mathrm{t}_{1} \Leftrightarrow \Leftrightarrow_{\text {def }}\left(\mathrm{t}_{1}<\mathrm{t}_{2} \wedge \forall \mathrm{t}_{3}\left(\mathrm{t}_{1}<\mathrm{t}_{3} \rightarrow\left(\mathrm{t}_{2} \leq \mathrm{t}_{3}\right)\right)\right)$

5. $\mathrm{t}_{2}=$ pred $\mathrm{t}_{1} \Leftrightarrow \Leftrightarrow_{\text {def }}$ succ $\mathrm{t}_{2}=\mathrm{t}_{1}$

A completely undetermined temporal instant is indicated by *.

6. $* \Leftrightarrow_{\mathrm{def}}$ during $[\infty, \infty]$

7. before $\mathrm{t} \Leftrightarrow{ }_{\text {def }}$ during $[\infty$, pred $\mathrm{t}]$

8. after $\mathrm{t} \Leftrightarrow{ }_{\mathrm{def}}$ during [succ $\left.\mathrm{t}, \infty\right]$

\subsection{Axiomatics}

The axioms of LITO are grouped in basic, modal, temporal and non-temporal axioms, according to their predicative symbols.

Basic axioms:

Basic axioms encompass the axioms of first-order logic plus the total order predicate $(\leq)$.

Modal axioms:

Modal axioms characterize the modalities Maybe $(M)$ and Known $(K)$, besides negation. Facts quantified by the $K$ operator represents the database and those with $M$ are derived possibilities.

1. $\forall \mathrm{x}(\mathrm{F} \vee \mathrm{G}) \rightarrow M \mathrm{~F}$

- Each component of a conjunction can be true.

2. $\forall \mathrm{x} \neg(K \neg \mathrm{F}) \rightarrow M \mathrm{~F}$

- Facts that are not known as false may be true (OWA). Non-temporal axioms:

Non-temporal axioms encompass only binary predicate $\mathrm{P}(\mathrm{x}, \mathrm{Tx})$ where $\mathrm{P}$ denotes a non-temporal attribute of an object.

3. $\forall \mathrm{x} \forall \mathrm{x}_{1} \forall \mathrm{x}_{2}\left(K \mathrm{P}\left(\mathrm{x}, \operatorname{or}\left(\mathrm{x}_{1}, \mathrm{x}_{2}\right)\right) \rightarrow M \mathrm{P}\left(\mathrm{x}, \mathrm{x}_{1}\right)\right)$

4. $\forall \mathrm{x} \forall \mathrm{x}_{1}\left(K \mathrm{P}(\mathrm{x}, *) \rightarrow \exists \mathrm{x}_{1} \mathrm{P}\left(\mathrm{x}, \mathrm{x}_{1}\right) \wedge \neg K \mathrm{P}\left(\mathrm{x}, \mathrm{x}_{1}\right)\right)$

5. $\forall \mathrm{x}(K \mathrm{P}(\mathrm{x}$, none $)) \rightarrow \forall \mathrm{x}_{1} \neg \mathrm{P}\left(\mathrm{x}, \mathrm{x}_{1}\right)$

Temporal axioms:

Temporal axioms include the predicates $\operatorname{Time}(\mathrm{x}, \mathrm{TI})$, i.e., predicates determining the time when an object is valid.

6. $\forall \mathrm{x} \forall \mathrm{I}(\operatorname{MTime}(\mathrm{x}, \mathrm{I}) \leftrightarrow \exists \mathrm{t}(\mathrm{t} \in \mathrm{I} \wedge \operatorname{Time}(\mathrm{x},[\mathrm{t}, \mathrm{t}]) \wedge$ 
$\neg \operatorname{KTime}(\mathrm{x},[\mathrm{t}, \mathrm{t}])))$

- The time of an object is possibly valid in the interval I, iff it is valid for at least an instant in I unknown by ITDB.

7. $\forall \mathrm{x} \forall \mathrm{I} \forall \mathrm{t}_{1}\left(\operatorname{Time}\left(\mathrm{x},\left[\right.\right.\right.$ during $\left.\left.\mathrm{I}, \mathrm{t}_{1}\right]\right) \rightarrow \exists \mathrm{t}(\mathrm{t} \in \mathrm{I} \wedge$ $\left.\operatorname{Time}\left(\mathrm{x},\left[\mathrm{t}, \mathrm{t}_{1}\right]\right) \wedge \neg \operatorname{KTime}\left(\mathrm{x},\left[\mathrm{t}, \mathrm{t}_{1}\right]\right)\right)$

- The interval represented by [during $\left.\mathrm{I}, \mathrm{t}_{1}\right]$ is valid starting from an I's initial instant $\mathrm{t}$ which is unknown by ITDB

8. $\forall \mathrm{x} \forall \mathrm{t}_{1} \forall \mathrm{I}$ (Time $\left(\mathrm{x},\left[\mathrm{t}_{1}\right.\right.$, during $\left.\left.\mathrm{I}\right]\right) \rightarrow \exists \mathrm{t}(\mathrm{t} \in \mathrm{I} \wedge$ $\left.\operatorname{Time}\left(\mathrm{x},\left[\mathrm{t}_{1}, \mathrm{t}\right]\right) \wedge \neg \operatorname{KTime}\left(\mathrm{x},\left[\mathrm{t}_{1}, \mathrm{t}\right]\right)\right)$

- The interval represented by [ $\mathrm{t} 1$, during $\mathrm{I}]$ is valid starting from an I's final instant $t$ which is unknown by ITDB.

\subsection{Rules}

In order to simplify our explanation, rules are categorized in basic rule and retrieval rules.

Basic rule:

1. if $\mid-\mathrm{F}$ and $\mid-\mathrm{F} \rightarrow \mathrm{G}$ then $\mid-\mathrm{G}$ (modus ponens)

Retrieval rules:

The rules for processing queries are given as Horn clauses or formulas reducible to Horn clauses. When a query is formulated the free variable of the head predicate should be instantiated [3]. The other variable is free and will contain the result of the rule's application, i.e., it will contain the answer.

Non-temporal retrieval rules:

These rules do not entail the retrieval of temporal values. The value of the argument $\mathrm{x}_{1}$, denoted by predicate $\mathrm{P}_{1}\left(\mathrm{x}, \mathrm{x}_{1}\right)$ of a query, can match completely or partially with a value of the same predicate in ITDB. The set of possible values of an undetermined attribute (for instance, salary ( $\mathrm{x}$, lt 1000$)$ is called the predicate's domain.

The matching is complete if both query and ITDB domains are precisely similar. Elsewhere, i.e., if there is an intersection between these domains, the matching is partial. If there is no intersection between these domains, the resulting value is the unknown value.

The second argument of the predicate $\mathrm{P}_{2}\left(\mathrm{x}, \mathrm{x}_{2}\right)$ specifies retrieval values from ITDB. These values are qualified by the truth value $M$ (maybe), $\neg$ (no) or yes (when a symbol is absent). For instance, the query "What is Mary's salary?" will be satisfied by the following rule:

employee $(\mathrm{x}$, Mary $) \wedge K$ employee $(\mathrm{x}$, Mary $) \wedge K$

$\operatorname{salary}\left(\mathrm{x}, \mathrm{x}_{2}\right) \rightarrow \operatorname{salary}\left(\mathrm{x}, \mathrm{x}_{2}\right)$

There are rules where the values of the queried argument $\mathrm{x}_{1}$ match completely, and there are rules where they match partially.

2. $\mathrm{P}_{1}\left(\mathrm{x}, \mathrm{x}_{1}\right) \wedge K \mathrm{P}_{1}\left(\mathrm{x}, \mathrm{x}_{1}\right) \wedge K \mathrm{P}_{2}\left(\mathrm{x}, \operatorname{or}\left(\mathrm{x}_{2}, \mathrm{x}_{3}\right)\right) \rightarrow M \mathrm{P}_{2}(\mathrm{x}$, $\left.\mathrm{x}_{2}\right)$

3. $\mathrm{P}_{1}\left(\mathrm{x}, \mathrm{x}_{1}\right) \wedge K \mathrm{P}_{1}\left(\mathrm{x}, \mathrm{x}_{1}\right) \wedge K \mathrm{P}_{2}(\mathrm{x}, *) \rightarrow M \mathrm{P}_{2}\left(\mathrm{x}, \mathrm{x}_{2}\right)$

4. $\mathrm{P}_{1}\left(\mathrm{x}, \mathrm{x}_{1}\right) \wedge K \mathrm{P}_{1}\left(\mathrm{x}, \operatorname{or}\left(\mathrm{x}_{1}, \mathrm{x}_{3}\right)\right) \wedge K \mathrm{P}_{2}\left(\mathrm{x}, \mathrm{x}_{2}\right) \rightarrow M \mathrm{P}_{2}(\mathrm{x}$, $\mathrm{x}_{2}$ )

5. $\mathrm{P}_{1}\left(\mathrm{x}, \mathrm{x}_{1}\right) \wedge K \mathrm{P}_{1}(\mathrm{x}, *) \wedge K \mathrm{P}_{2}\left(\mathrm{x}, \mathrm{x}_{2}\right) \rightarrow M \mathrm{P}_{2}\left(\mathrm{x}, \mathrm{x}_{2}\right)$

Temporal retrieval rules:

These rules entail only the retrieval of temporal values. The answers of a query depend of the relation between the times of the query predicates and the ITDB predicates.

6. $\operatorname{Time}\left(\mathrm{x}, \mathrm{I}_{1}\right) \wedge K \mathrm{P}\left(\mathrm{x}, \mathrm{x}_{1}\right) \wedge \operatorname{KTime}\left(\mathrm{x}, \mathrm{I}_{2}\right) \wedge \mathrm{I}_{1} \subseteq \mathrm{I}_{2} \rightarrow$ $\mathrm{P}\left(\mathrm{x}, \mathrm{x}_{1}\right)$

7. $\operatorname{Time}\left(\mathrm{x}, \mathrm{I}_{1}\right) \wedge K \mathrm{P}\left(\mathrm{x}, \mathrm{x}_{1}\right) \wedge \operatorname{KMTime}\left(\mathrm{x}, \mathrm{I}_{2}\right) \wedge \mathrm{I}_{1} \subseteq \mathrm{I}_{2} \rightarrow$ $M \mathrm{P}\left(\mathrm{x}, \mathrm{x}_{1}\right)$

When the object's time is queried, the true value returned will be determined by the precision in Time predicate and/or in the non-temporal predicate.

8. $\mathrm{P}\left(\mathrm{x}, \mathrm{x}_{1}\right) \wedge K \mathrm{P}\left(\mathrm{x}, \mathrm{x}_{1}\right) \wedge \operatorname{KTime}(\mathrm{x}, \mathrm{I}) \rightarrow \operatorname{Time}(\mathrm{x}, \mathrm{I})$

9. $\mathrm{P}\left(\mathrm{x}, \mathrm{x}_{1}\right) \wedge K \mathrm{P}\left(\mathrm{x}, \mathrm{x}_{1}\right) \wedge \operatorname{KMTime}(\mathrm{x}, \mathrm{I}) \rightarrow \operatorname{MTime}(\mathrm{x}, \mathrm{I})$

\subsection{Modifying the database}

Whereas updating a conventional database inserts and deletes objects or changes the values of attributes, in a system with imprecisions, the incoming new information could mean a reduction of the imprecision instead of an update removing old values. Note that in our ITDB an unary predicate $\mathrm{P}(\mathrm{x})$ identifies an objects and a binary predicates $\mathrm{P}(\mathrm{x}, \mathrm{y})$ determines a value $\mathrm{y}$ of object $\mathrm{x}$. The $\mathrm{x}$ denotes a pair $<$ oid, $t>$ which identifies the object and the transaction time when it was created.

We distinguish 4 modifying operations: insert, delete, refine, and update. Insert, delete and update are as for conventional database, considering conventions for temporal databases, where old values are kept in the database with closed valid-time intervals. The other difference is that the value y could be imprecise. With the refine operator the value y should be imprecise and the result is the intersection of the imprecisions of the new data and the database. For instance, if we have a fact salary (o2, lt 2000) the application of refine(salary(o2, gt 1000)) results in $\operatorname{salary}(02$, in $(1000,2000))$. The same applies to the temporal attributes.

\subsection{Application}

We illustrate the application of the logic with a simple example. Assume a class of objects of type employment(employee, company, time) containing objects $\mathrm{O} 1$ and $\mathrm{O} 2$, represented as follows:

employment $(\mathrm{O} 1) \wedge$ employee $(\mathrm{O} 1$, or $($ Peter, Mary $)) \wedge$ company $(\mathrm{O} 1$, or $(\mathrm{UFPE}, \mathrm{UFPB})) \wedge \operatorname{time}\left(\mathrm{O} 1,\left[1 / 1 / 1994,{ }^{*}\right]\right)$ 
employment $(\mathrm{O} 2) \wedge$ employee $(\mathrm{O} 2, \operatorname{not}($ Mary $)) \wedge$ company $(\mathrm{O} 2$, (UFC) $\wedge$ time $(\mathrm{O} 2$, [before 01/01/1983, now])

The object $\mathrm{O} 1$ represents an employment of a person, which is Peter or Mary, working for UFPE or UFPB since $1 / 1 / 1994$ up to an unknown date. O2 is an employment of a person different from Maria, working at UFC since before 1983 .

The query "does Mary work for UFPB at 1/1/2001?" is formalized as

employee $(\mathrm{x}$, Mary $) \wedge \operatorname{company}(\mathrm{x}, \mathrm{UFPB}) \wedge \operatorname{time}(\mathrm{x}$, $1 / 1 / 2001)$

So, since company(x, UFPB) matches partially with rule 4, the predicate employee ( $\mathrm{x}$, Mary) of the query is instantiated in this rule together with the facts of the database and results

employee $(\mathrm{O} 1$, Mary) $\wedge$ Kemployee $(\mathrm{O} 1$, or(Peter, Mary $))$

$\wedge$ Kcompany $(\mathrm{O} 1, \mathrm{UFPB}) \rightarrow \operatorname{Mcompany}(\mathrm{O} 1, \mathrm{UFPB})$

The same reasoning can be applied to rule 9 resulting employee $(\mathrm{O} 1$, Mary $) \wedge$ Kcompany $(\mathrm{O} 1, \mathrm{UFPB}) \wedge$ KMTime $(\mathrm{O} 1,1 / 1 / 2001) \rightarrow \operatorname{MTime}(\mathrm{O} 1,1 / 1 / 2001)$

Combining these results and interpreting $M$ as a possibility, we get the final answer possible.

\section{Final Remarks}

This paper suggests a formalism that contains complete definitions about representation and manipulation of objects in both ITDB and temporal database. New forms were proposed to represent the nontemporal and temporal indeterminacy present in these objects. Also, conception and construction of mechanisms for logic queries, retrieval queries, insertions, logic deletions, refinements and updates in these databases were elaborated. All these new characteristics make this formalism a model to treat indeterminate temporal objects.

The logic presented here is the formal basis for a system, called MITO-Management of Incomplete Temporal Objects [1,2], which was implemented in LPAProlog [9] and shows how an application with temporal indeterminate objects could be queried and updated.

\section{References}

[1] Bezerra E., "Representation and Manipulation of Objects in Indeterminate Temporal Databases", Ph. D. thesis, Federal University of Paraiba, 2000

[2] Bezerra E., Schiel U. and Lula Jr. B. "Towards a Logic For Representation and Retrieval of Incomplete Temporal Objects", Proc. of $1^{\text {st }}$. International Workshop on Computer
Science and Information Technologies, CSIT'99, Moscow, Russia, 1999

[3] Böhlen M., Chomicki J., Snodgrass R. and Toman D. "Querying TSQL2 Databases with Temporal Logic", Apers P., Bouzeghoub M. and Gardarin G. (Ed.), Advances in Database Technology: EDBT'96, Springer-Verlag, pages 325341, 1996

[4] Dyreson C. and Snodgrass R. "Suporting Valid-time Indeterminacy", ACM Trans. on Database Systems, volume 23, number 1, pages 1-57, 1998

[5] Etzion O., Jajodia S. and Sripada S. Temporal Databases: Research and Practice, Springer, 1998

[6] Hughes G. and Cresswell M. A New Introduction to Modal Logic, Routledge, 1996

[7] Jensen C.S. and Dyreson C.E. (eds.). "A Consensus Glossary of Temporal Database Concepts - February 1998 Version", in [5], pages 367-405, 1998

[8] Keller A. and Wilkins M. "On the Use of an Extended Relational Model to Handle Changing Incomplete Information", IEEE Transactions on Software Engineering, number 7, 1985

[9] LPA WIN-PROLOG 3.5. Win32 Programming Guide, 1997

[10] Levesque H. "The Logic of Incomplete Knowledge Bases", Mylopoulos J, Brodie M. (eds) Readings of Artificial Intelligence \& Database. Morgan Kaufmann Publishers, pages 328-341, 1994

[11] Lipski W. "On Semantic Considerations Connected with Incomplete Information Databases", ACM TODS vol.4, pages 262-296, 1979

[12] Melton J. (ed.). (ISO Working Draft SQL/Temporal), ANSI X3H2-97-135, ISO DBL:LGW-013, April, 1997

[14] Snodgrass R. (ed.). The TSQL2 Temporal Query Language, Kluwer Academic Publishers, 1995. 